\title{
PULMONARY SECRETORY PHOSPHOLIPASE A2 IS PRODUCED ALONG WITH CLARA PROTEIN IN HUMAN NEONATES WITH MECONIUM ASPIRATION AND CORRELATES WITH OXYGENATION IMPAIRMENT AND EPITHELIAL DAMAGE
}

\author{
D. De Luca ${ }^{1,2}$, V.P. Carnielli ${ }^{2}$, A. Minucci ${ }^{3}$, D. Tripodi ${ }^{3}$, M. Piastra ${ }^{1}$, D. Pietrini ${ }^{1}$, E.D. Capoluongo ${ }^{3}$, G. \\ Conti $^{1}$ \\ ${ }^{1}$ PICU, University Hospital 'A.Gemelli', Catholic University of the Sacred Heart, Roma, ${ }^{2}$ NICU, \\ Polytechnical University of Marche, Ancona, ' Clinical Molecular Biology, University Hospital 'A.Gemelli', \\ Catholic University of the Sacred Heart, Roma, Italy
}

Background: Meconium aspiration syndrome (MAS) is a life-threatening lung injury mainly studied in animal models. From these it is known that pancreatic secretory phospholipase A2 (sPLA2-IB) and proinflammatory cytokines present in meconium challenge the lungs, catabolising surfactant and harming the alveolar epithelium. Locally produced phospholipases could perpetuate the injury and have consequences on the clinical pictures. It is unknown whether pulmonary phospholipases A2 (sPLA2-IIA) is involved.

Methods: We measured the distinct phospholipases A2 and their modulators in broncho-alveolar lavage fluids and in meconium, using specific ELISA/EIA kits. 5 neonates affected by MAS and 5 control age/weight matched neonates were enrolled. Findings were corrected for urea ratio or for dilutional factors.

Results: MAS patients have higher amount of pulmonary phospholipase (sPLA2-IIA; $p=0.016$ ) and Clara Cell Secretory Protein (CCSP; $p=0.032$ ). The local lung production of such proteins is confirmed by their low levels in meconium and pulmonary sPLA2-IIA contributes to the higher total enzyme activity in MAS patients, as compared to controls $(p=0.008)$. Cytosolic phospholipase A2 was always undetectable. Correlations showed that enzyme total activity and sPLA2-IIA concentrations are linked with the TNF $\alpha$ present in meconium and that an increase in phospholipase was associated with the release of CCSP. Phospholipase global activity, sPLA2-IIA and TNF $\alpha$ in broncho-alveolar lavage fluids correlate with oxygenation impairment and haemorrhagic lung oedema.

Conclusions: Pulmonary sPLA2 (and not only the pancreatic isotype) plays a role in the oxygenation impairment and epithelial damage during MAS. This may be a target to be addressed with sPLA2 inhibitors. 